Scientia Marina 71(1)

March 2007, 75-86, Barcelona (Spain)

ISSN: 0214-8358

\title{
A review of the Tripterygion tripteronotus (Risso, 1810) complex, with a description of a new species from the Mediterranean Sea (Teleostei: Tripterygiidae)
}

\author{
JOSEP CARRERAS-CARBONELL ${ }^{1}$, MARTA PASCUAL ${ }^{2}$ and ENRIQUE MACPHERSON ${ }^{1}$ \\ ${ }^{1}$ Centre d'Estudis Avançats de Blanes (CSIC), Carr. Acc. Cala St. Francesc 14, Blanes, 17300 Girona, Spain. \\ E-mail: pep@ceab.csic.es \\ ${ }^{2}$ Dept. Genètica, Univ. Barcelona, 08028 Barcelona, Spain.
}

\begin{abstract}
SUMMARY: We compared specimens of Tripterygion tripteronotus from 52 localities of the Mediterranean Sea and adjacent waters, using four gene sequences (12S rRNA, tRNA-valine, 16S rRNA and COI) and morphological characters. Two well-differentiated clades with a mean genetic divergence of $6.89 \pm 0.73 \%$ were found with molecular data, indicating the existence of two different species. These two species have disjunctive geographic distribution areas without any molecular hybrid populations. Subtle but diagnostic morphological differences were also present between the two species. T. tripteronotus is restricted to the northern Mediterranean basin, from the NE coast of Spain to Greece and Turkey, including the islands of Malta and Cyprus. T. tartessicum n. sp. is geographically distributed along the southern coast of Spain, from Cape of La Nao to the Gulf of Cadiz, the Balearic Islands and northern Africa, from Morocco to Tunisia. According to molecular data, these two species could have diverged during the Pliocene glaciations 2.7-3.6 Mya.
\end{abstract}

Keywords: Tripterygion, new species, molecular data, morphology, taxonomy, Mediterranean Sea.

RESUMEN: REVISIÓN DEL COMPLEJO TRIPTERYGION TRIPTERONOTUS (RISSO, 1810), Y DESCRIPCIÓN DE UNA NUEVA ESPECIE EN EL MAR MEDITERRÁNEO (TELEOSTEI: TRIPTERYGIIDAE). - Se han estudiado especímenes de Tripterygion tripteronotus procedentes de 52 localidades mediterráneas y de aguas atlánticas adyacentes, utilizando cuatro genes mitocondriales distintos (12S rRNA, tRNA-valine, 16S rRNA y COI) así como varios caracteres morfológicos. Se han encontrado dos grupos molecularmente bien diferenciados, la divergencia genética media presente entre ambos es de un $6.89 \pm 0.73 \%$, lo que implica la presencia de dos especies distintas. Sus áreas de distribución están separadas y no se han encontrado poblaciones molecularmente híbridas. Además, se han encontrado pequeñas diferencias morfológicas que pueden ser utilizadas como caracteres diagnósticos entre las dos especies. T. tripteronotus se encuentra en la cuenca mediterránea norte, extendiéndose desde la costa NE de España hasta Grecia y Turquía, incluyendo las islas de Malta y Chipre. T. tartessicum n. sp. se extiende por la costa sur de España, desde Cabo La Nao hasta el Golfo de Cádiz, las islas Baleares y el norte de Africa, desde Marruecos a Túnez. De acuerdo con los datos moleculares obtenidos, ambas especies pudieron divergir durante las glaciaciones ocurridas en el Plioceno hace unos 2.7-3.6 m.a.

Palabras clave: Tripterygion, nueva especie, datos moleculares, morfología, taxonomía, Mar Mediterráneo.

\section{INTRODUCTION}

Molecular data provide a complementary approach to discriminate species separated by subtle morphological characters (Knowlton, 1993; Avise, 1994; Held and Wagele, 2005). In the last few years, numerous authors have used molecular methods to detect cryptic species, either in fishes (Gilles et al., 2000; Gysels et al., 2004; Almada et al., 2005a) or in other marine organisms (Tarjuelo et al., 2001).

The family Tripterygiidae contains species of bottom-living blennioid fishes, usually associated with rocky habitats and inhabiting cold, temperate, subtropical and tropical areas (Fricke, 2002). The 
genus Tripterygion Risso, 1826, is the only genus of the family Tripterygiidae in the Mediterranean Sea and on the northeastern Atlantic coast (Zander, 1986). Three species have been described: $T$. tripteronotus, Risso, 1810, and T. melanurus, Guichenot, 1845, are endemic to the Mediterranean, and T. delaisi Cadenat and Blache, 1971, is found in both areas (Wirtz, 1980). Individuals of the three species are common in shallow coastal waters, always living in rocky areas. T. tripteronotus inhabits light-exposed and shady biotopes preferably between 0 and $3 \mathrm{~m}$, whereas $T$. delaisi uses similar biotopes but at greater depth (between 0 and $40 \mathrm{~m}$ ) and also biotopes with reduced light such as under overhanging rocks or entrances to caves. Finally, $T$. melanurus inhabits walls or ceilings of marine caves and other dimly lit biotopes (Wirtz, 1978; Macpherson, 1994; Zander, 2004).

The species of the genus Tripterygion form a monophyletic group and each previously described species is well differentiated genetically (CarrerasCarbonell et al., 2005). However, this recent phylogeographic study, using molecular data, indicated that: (1) the two morphotypes of T. melanurus, traditionally considered as two different subspecies by Zander (1986), were not genetically different with the markers used, although there may be differences on other parts of the DNA sequence, (2) the two currently accepted subspecies for T. delaisi (Zander, 1986) were molecularly validated, and (3) $T$. tripteronotus, considered at present as a single species, showed two well-defined and highly supported clades with greater divergence than that shown between the two T. delaisi subspecies, revealing the existence of two cryptic species within $T$. tripteronotus (Carreras-Carbonell et al., 2005). Zander and Heymer (1970) had already described two different pattern bands in the caudal region for T. tripteronotus individuals from Banyuls-sur-Mer (France) and Mdiq (Morocco). Later, Zander and Heymer (1976) showed slight morphological differences in the dorsal fins between $T$. tripteronotus specimens from Israel and Lebanon in comparison with specimens from the northwestern Mediterranean. Although no taxonomic status was assigned, these morphological differences could be related to the two T. tripteronotus clades found by Carreras-Carbonell et al. (2005).

The aim of the present work was to describe the new species and search for morphological characters that allow the two species to be differentiated using specimens from 52 localities of the Mediterranean Sea and adjacent waters.

\section{MATERIALS AND METHODS}

\section{Sampling and repositories}

Specimens of the two species of Tripterygion were collected at different localities of the Mediterranean Sea and the Gulf of Cadiz; specimens from the Staatliches Museum fuer Naturkunde (Stuttgart, SMNS) were also used, with the result that the individuals came from a total of 52 localities (Fig. 1). The number of individuals used for morphological and molecular analyses, as well as supplementary details about each sampling locality, are shown in Table 1.

The type series of the new species are deposited in the collections of the Instituto de Ciencias del Mar (Barcelona, IIPB), the Museo Nacional de Ciencias Naturales (Madrid, MNCN) and the Staatliches Museum fuer Naturkunde (Stuttgart, SMNS) (see Table 1).

\section{Morphological analysis}

In the description of the new species, the data of the paratypes follow those of the holotype, in parentheses. Lengths given and the terminology and other measurements used mainly follow Zander and Heymer (1970), Wheeler and Dunne (1975) and Fricke (1997). Lengths are explained below:

Predorsal length (PD) distance between middle of upper lip and base of the $1^{\text {st }}$ spine of the first dorsal fin.

Head length (HL) distance between middle of upper lip and upper insertion of operculum.

Orbital diameter (OD) maximum eye diameter.

Preorbital length (PO) distance between middle of upper lip and anterior margin of eye.

The middle of the upper lip is used as the starting point for several lengths rather than the tip of the upper jaw, as the latter may be protractile.

Mandibular pore formula. This formula gives the number of pores under left dentary + number of median pore $(\mathrm{s})+$ number of pores under right dentary.

Individuals were photographed alive in order to check their colour pattern; one or two right gills were removed and kept in absolute ethanol at room temperature. Specimens were individually fixed using buffered formol with $2 \%$ borax to maintain the colour pattern for further morphological analyses. 


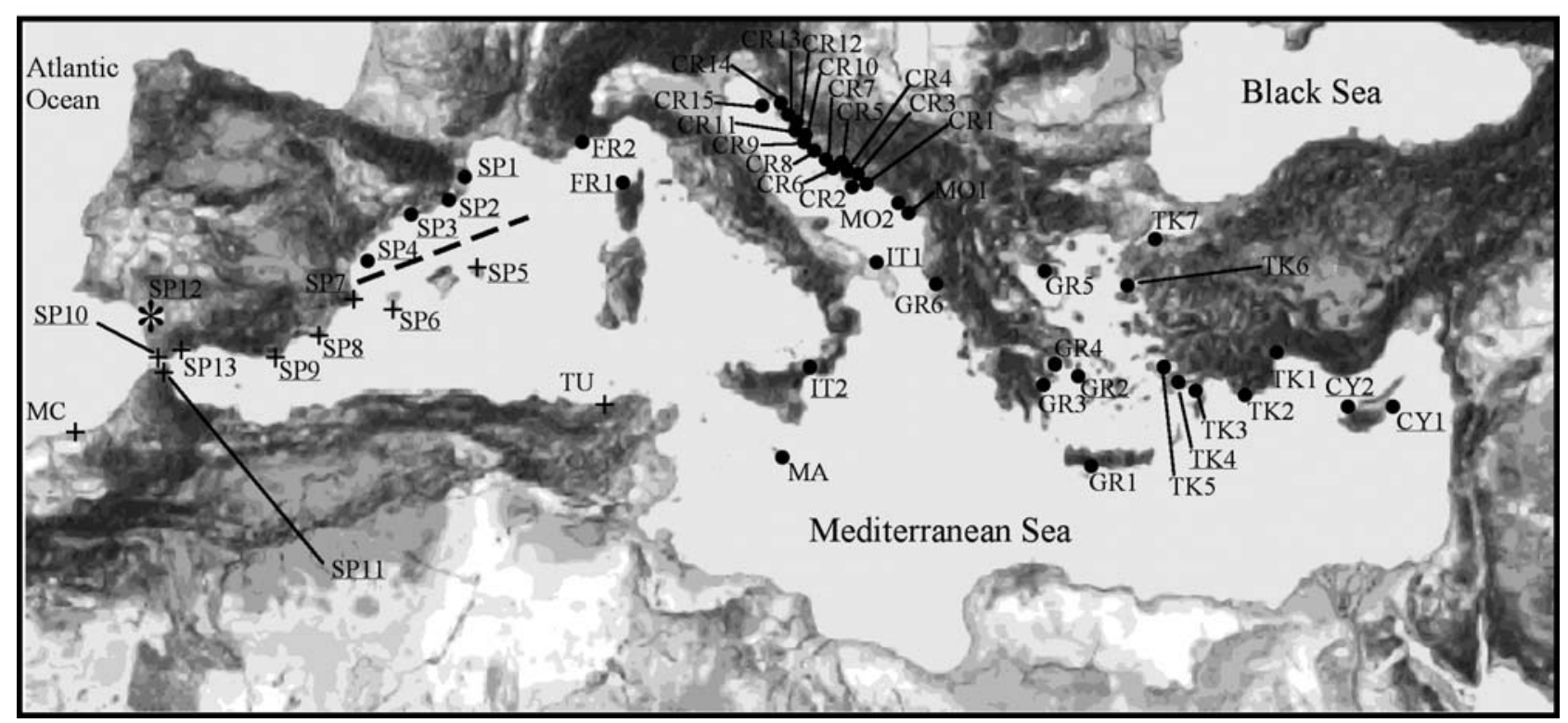

FIG. 1. - Sampling localities for T.tripteronotus $(\bullet)$ and T. tartessicum (+). Dashed line shows the break zone between the two species along the Spanish Mediterranean coast. (*): Holotype locality. Localities which individuals were molecularly analysed are underlined. See Table 1 for locality abbreviations and further details.

Key

The morphological taxonomic key only works for both sexes when morphometric measurements are used. Sometimes, males can also be distinguished by discrete morphological characters, while females are identifiable only by their geographical distribution and accompanying males.

\section{Molecular analysis}

In order to analyse the genetic difference between the two clades of T. tripteronotus, we used the sequences of the 12S, tRNA-valine and $16 \mathrm{~S}$ (acc. num: AJ868510-23, AJ937970-74, AJ87214960 and AJ937975-79), and COI (acc. num: AJ872128-40 and AJ937862-65) genes from Carreras-Carbonell et al. (2005). The same gene sequences were amplified from additional individuals from CY1 (AM260942 and AM260946), CY2 (AM260943 and AM260946), TK4 (AM260944 and AM260947), TK6 (AM260944 and AM260947), IT2 (AM260940-1 and AM260945), FR2 (AM086386-7), SP3 (AM086388-9), SP4 (AM086390-1), $\quad$ SP5 (AM086392-3), SP6 (AM086394-5), SP7 (AM086396-7) and SP8 (AM086398-9) (for location abbreviations and further sampling locality details see Table 1). We used Tripterygion delaisi xanthosoma (family Tripterygiidae) and Parablennius rouxi (family Blenniidae) from SP2 as internal and external out- group species respectively (AJ868503, AJ872118 and AJ872164 for T. d. xanthosoma and AJ96665662 for P. rouxi).

The homogeneity of base composition across taxa was assessed using the goodness-of-fit $\left(\chi^{2}\right)$ test and the incongruence length difference test (ILD) (Farris et al., 1994) was computed to assess analytical differences between genes; both tests are implemented in PAUP* ver. 4.0b10 (Swofford, 2001). In the latter test only parsimony informative characters were included and heuristic searches were performed with 10 random stepwise additions with TBR branch swapping and 1000 randomisations. Furthermore, trees were considered significantly incongruent whenever different gene trees conflicted at nodes that were supported by BI posterior probabilities $>95 \%$ (Moyer et al., 2004).

Phylogenetic trees were inferred by Bayesian inference (BI) using Mr Bayes 3.0b4 (Huelsenbeck and Ronquist, 2001) because it seems to be the best methodology for inferring phylogenetic relationships between species (Alfaro et al., 2003), and its reconstruction does not seem to be affected by saturated positions (Carreras-Carbonell et al., 2005). The computer program MODELTEST ver. 3.06 (Posada and Crandall, 1998) was used to choose the best-fit ML model under the Akaike Information Criterion (AIC) for each gene separately and was later applied in the BI analyses. The MCMC (Markov chain Monte Carlo) algorithm with four Markov chains was run for 1500000 generations, sampled every 100 generations 
TABLE 1. - Specimens of the two Tripterygion species collected at different localities of the Mediterranean and Atlantic adjacent waters. The number of individuals used for morphological $(\mathrm{Nm})$ and molecular $(\mathrm{Ng})$ analyses, for each locality, are detailed.

\begin{tabular}{|c|c|c|c|c|c|c|c|c|c|}
\hline & Map code & Country & Locality & $\begin{array}{l}\text { Latitude/ } \\
\text { Longitude }\end{array}$ & $\begin{array}{l}\text { Depth } \\
(\mathrm{m})\end{array}$ & $\begin{array}{l}\text { Collection } \\
\text { date }\end{array}$ & $\begin{array}{c}\mathrm{Nm} / \\
\mathrm{Ng}\end{array}$ & $\begin{array}{l}\text { LT range } \\
(\mathrm{mm})\end{array}$ & $\begin{array}{l}\text { Catalogue } \\
\text { number }\end{array}$ \\
\hline \multirow{34}{*}{$\begin{array}{l}\text { Tripterygion } \\
\text { tripteronotus }\end{array}$} & CY1 & Cyprus & Akrotirion Gatas/Cape & $34^{\circ} 32^{\prime} \mathrm{N}$ & $0-1$ & May 2002 & $6 / 4$ & $42-47$ & SMNS \\
\hline & & & Greco, southeastern corner & $33^{\circ} 00^{\prime} \mathrm{E}$ & & & & & 23059 \\
\hline & CY2a & Cyprus & Karavas Alsavcak Bay, small & $35^{\circ} 21^{\prime} 13^{\prime \prime} \mathrm{N}$ & $0-1$ & 20 May 1997 & $1 / 0$ & 38 & SMNS \\
\hline & & & $\begin{array}{l}\text { island on eastern side of bay, } 9 \mathrm{~km} \\
\text { west of Kyrenia/Kyreneia/Girne }\end{array}$ & $33^{\circ} 13^{\prime} 15^{\prime \prime} \mathrm{E}$ & & & & & 19066 \\
\hline & CY2b & Cyprus & $\begin{array}{l}\text { Karavas Alsavcak Bay, small } \\
\text { island on eastern side of bay, } 9 \mathrm{~km}\end{array}$ & $\begin{array}{l}35^{\circ} 21^{\prime} 13^{\prime}, \mathrm{N} \\
33^{\circ} 13^{\prime} 06^{\prime} \mathrm{E}\end{array}$ & $0-1$ & 23 May 1997 & $1 / 0$ & 41 & $\begin{array}{l}\text { SMNS } \\
19085\end{array}$ \\
\hline & CY2c & Cyprus & $\begin{array}{l}\text { west of Kyrenia/Kyreneia/Girne } \\
\text { Karavas Alsavcak Bay, small } \\
\text { island on eastern side of bay, } 9 \mathrm{~km}\end{array}$ & $\begin{array}{l}35^{\circ} 21^{\prime} 13^{\prime \prime}, \mathrm{N} \\
33^{\circ} 13^{\prime} 06^{\prime} \mathrm{E}\end{array}$ & $0-1$ & 24 May 1997 & $5 / 1$ & $34-43$ & $\begin{array}{l}\text { SMNS } \\
19091\end{array}$ \\
\hline & CY2d & Cyprus & $\begin{array}{l}\text { west of Kyrenia/Kyreneia/Girne } \\
\text { Karavas Alsavcak Bay, small } \\
\text { island on eastern side of bay, } 9 \mathrm{~km}\end{array}$ & $\begin{array}{l}35^{\circ} 21^{\prime} 13^{\prime \prime} \mathrm{N} \\
33^{\circ} 13^{\prime} 06^{\prime} \mathrm{E}\end{array}$ & $0.6-1.5$ & 27 May 1997 & $6 / 2$ & $36-43$ & $\begin{array}{l}\text { SMNS } \\
19098\end{array}$ \\
\hline & CY2e & Cyprus & $\begin{array}{l}\text { west of Kyrenia/Kyreneia/Girne } \\
\text { Karavas Alsavcak Bay, small } \\
\text { island on eastern side of bay, } 9 \mathrm{~km} \\
\text { west of Kyrenia/Kyreneia/Girne }\end{array}$ & $\begin{array}{l}35^{\circ} 21^{\prime} 13^{\prime \prime} \mathrm{N} \\
33^{\circ} 13^{\prime} 06^{\prime} \mathrm{E}\end{array}$ & $3-5.5$ & 27 May 1997 & $1 / 0$ & 37 & $\begin{array}{l}\text { SMNS } \\
19106\end{array}$ \\
\hline & CY2f & Cyprus & $\begin{array}{l}\text { Karavas Alsavcak Bay, rocky shore } \\
\text { and cave on western side of bay, }\end{array}$ & $\begin{array}{l}35^{\circ} 21^{\prime} 13^{\prime \prime} \mathrm{N} \\
33^{\circ} 13^{\prime} 06^{\prime} \mathrm{E}\end{array}$ & $0-1$ & 23 May 1997 & $2 / 1$ & $36-41$ & $\begin{array}{l}\text { SMNS } \\
19089\end{array}$ \\
\hline & CY2g & Cyprus & $\begin{array}{c}9 \mathrm{~km} \text { west of Kyrenia/Kyreneia/Girne } \\
\text { Karavas Alsavcak Bay, } 9 \mathrm{~km} \\
\text { west of Kyrenia/Kyreneia/Girne }\end{array}$ & $\begin{array}{l}35^{\circ} 21^{\prime} 12^{\prime \prime}, \mathrm{N} \\
33^{\circ} 13^{\prime} 07^{\prime \prime} \mathrm{E}\end{array}$ & $0-1$ & 22 May 1997 & $2 / 0$ & $38-42$ & $\begin{array}{l}\text { SMNS } \\
19089\end{array}$ \\
\hline & CY2h & Cyprus & Karavas Alsavcak Bay, small & $35^{\circ} 21^{\prime} 13^{\prime}, \mathrm{N}$ & $0-1$ & 18 May 1997 & $2 / 0$ & $34-37$ & SMNS \\
\hline & CY2i & Cyprus & $\begin{array}{l}\text { 1sland on eastern side of bay, } 9 \mathrm{~km} \\
\text { west of Kyrenia/Kyreneia/Girne } \\
\text { Karavas Alsavcak Bay, small } \\
\text { island on eastern side of bay, } 9 \mathrm{~km} \\
\text { west of Kyrenia/Kyreneia/Girne }\end{array}$ & $\begin{array}{l}35^{\circ} 21^{\prime} 13^{\prime}, \mathrm{N} \\
33^{\circ} 13^{\prime} 15^{\prime}, \mathrm{E}\end{array}$ & $0-1$ & 19 May 1997 & $4 / 1$ & $37-44$ & $\begin{array}{l}\text { SMNS } \\
19059\end{array}$ \\
\hline & TK1 & Turkey & Side, Pamphylia & $\begin{array}{l}36^{\circ} 45^{\prime} 58^{\prime \prime}, \mathrm{N} \\
31^{\circ} 23^{\prime} 04^{\prime \prime} \mathrm{E}\end{array}$ & n.a. & 5 June 1988 & $1 / 0$ & 43 & $\begin{array}{l}\text { SMNS } \\
8402\end{array}$ \\
\hline & TK2a & Turkey & Kas, Lycia, Antalya Province & $\begin{array}{l}36^{\circ} 11^{\prime} 30^{\prime}, \mathrm{N} \\
29^{\circ} 38^{\prime} 33^{\prime} \mathrm{E}\end{array}$ & n.a. & 9 June 1988 & $3 / 0$ & $36-42$ & $\begin{array}{l}\text { SMNS } \\
8408\end{array}$ \\
\hline & TK2b & Turkey & $\begin{array}{l}\text { Kas, southern harbour jetty, } \\
\text { Lycia, Antalya Province }\end{array}$ & $\begin{array}{l}36^{\circ} 11^{\prime} 46^{\prime}, \mathrm{N} \\
29^{\circ} 38^{\prime} 33^{\prime}, \mathrm{E}\end{array}$ & n.a. & 7 June 1988 & $2 / 0$ & $40-42$ & $\begin{array}{l}\text { SMNS } \\
8406\end{array}$ \\
\hline & TK2c & Turkey & Kas, Lycia, Antalya Province & $\begin{array}{l}36^{\circ} 11^{\prime} 30^{\prime \prime}, \mathrm{N} \\
29^{\circ} 38^{\prime} 33^{\prime \prime} \mathrm{E}\end{array}$ & n.a. & 10 June 1988 & $2 / 0$ & $42-45$ & $\begin{array}{l}\text { SMNS } \\
8407\end{array}$ \\
\hline & TK2d & Turkey & Kas, Lycia, Antalya Province & $\begin{array}{l}36^{\circ} 11,30^{\prime},{ }^{\prime} \\
29^{\circ} 38^{\prime} 33^{\prime}, \mathrm{E}\end{array}$ & n.a. & 11 June 1988 & $6 / 0$ & $38-47$ & $\begin{array}{l}\text { SMNS } \\
8389\end{array}$ \\
\hline & TK3 & Turkey & $\begin{array}{c}\text { Torba, ca. } 12 \text { km north of Bodrum, } \\
\text { Karia }\end{array}$ & $\begin{array}{l}37^{\circ} 07^{\prime} 24^{\prime}, \mathrm{N} \\
27^{\circ} 23^{\prime} 47^{\prime}, \mathrm{E}\end{array}$ & n.a. & 19 June 1988 & $7 / 0$ & $35-50$ & $\begin{array}{l}\text { SMNS } \\
8373\end{array}$ \\
\hline & TK4a & Turkey & Bodrum, Karia, Egean Sea & $\begin{array}{l}37^{\circ} 01,53,{ }^{\prime}, \mathrm{N} \\
27^{\circ} 25,38^{\prime}, \mathrm{E}\end{array}$ & n.a. & 16 June 1988 & $5 / 1$ & $39-58$ & $\begin{array}{l}\text { SMNS } \\
8392\end{array}$ \\
\hline & TK4b & Turkey & Bodrum, Karia, Egean Sea & $\begin{array}{l}37^{\circ} 011^{\prime}, 53, ' \mathrm{~N} \\
27^{\circ} 25^{\prime} 38^{\prime}, \mathrm{E}\end{array}$ & n.a. & 17 June 1988 & $8 / 2$ & $35-50$ & $\begin{array}{l}\text { SMNS } \\
8390\end{array}$ \\
\hline & TK5 & Turkey & Orag Island, Karia & $\begin{array}{l}36^{\circ} 58^{\prime}, 35^{\prime,}, \mathrm{N} \\
27^{\circ} 35^{\prime} 39^{\prime} \mathrm{E}\end{array}$ & n.a. & 29 June 1988 & $6 / 0$ & $35-52$ & $\begin{array}{l}\text { SMNS } \\
8375\end{array}$ \\
\hline & TK6 & Turkey & $\begin{array}{l}\text { Bay south of Ayvalik, } \\
\text { Province Balikesir, Egean Sea }\end{array}$ & $\begin{array}{l}39^{\circ} 14^{\prime} \mathrm{N} \\
26^{\circ} 38^{\prime} \mathrm{E}\end{array}$ & n.a. & 3 June 1969 & $1 / 1$ & 58 & $\begin{array}{l}\text { SMNS } \\
13607\end{array}$ \\
\hline & TK7 & Turkey & $\begin{array}{c}\text { Erdek, west of Bandirma, } \\
\text { Marmara Sea }\end{array}$ & $\begin{array}{l}40^{\circ} 24^{\prime} \mathrm{N} \\
27^{\circ} 48^{\prime} \mathrm{E}\end{array}$ & n.a. & 28 May 1969 & $10 /-$ & $40-59$ & $\begin{array}{l}\text { SMNS } \\
14326\end{array}$ \\
\hline & GR1a & Greece & Elounda, north of Aghios & $35^{\circ} 24^{\prime} \mathrm{N}$ & n.a. & 12 Aug. 1971 & $4 /-$ & $42-50$ & SMNS \\
\hline & GR1b & Greece & $\begin{array}{l}\text { Nikolaos, Kreta/Crete Island } \\
\text { Elounda, north of Aghios } \\
\text { Nikolaos, Kreta/Crete Island }\end{array}$ & $\begin{array}{l}24^{\circ} 40^{\prime} \mathrm{E} \\
35^{\circ} 24^{\prime} \mathrm{N} \\
24^{\circ} 40^{\prime} \mathrm{E}\end{array}$ & n.a. & 10 Aug. 1971 & $3 /-$ & $42-50$ & $\begin{array}{l}14371 \\
\text { SMNS } \\
14369\end{array}$ \\
\hline & GR2 & Greece & $\begin{array}{l}\text { Ciclades Is. } \\
\text { Kythnos Is. }\end{array}$ & $\begin{array}{l}36^{\circ} 43,35^{\prime}, \mathrm{N} \\
25^{\circ} 16^{\prime} 35^{\prime}, \mathrm{E}\end{array}$ & $0-2$ & 24 Oct. 2004 & $2 / 2$ & $42-55$ & IIPB \\
\hline & GR3 & Greece & Kyra Island, Gulf of Epidavros & $\begin{array}{l}37^{\circ} 37^{\prime} 30^{\prime}, \mathrm{N} \\
23^{\circ} 12^{\prime} 00^{\prime \prime} \mathrm{E}\end{array}$ & n.a. & 20 July 1970 & $7 /-$ & $28-36$ & $\begin{array}{l}\text { SMNS } \\
14361\end{array}$ \\
\hline & GR4 & Greece & $\begin{array}{l}\text { Aiyina Island, southern tip, } \\
\text { Saronian Gulf }\end{array}$ & $\begin{array}{l}37^{\circ} 41^{\prime} \mathrm{N} \\
23^{\circ} 24^{\prime} \mathrm{E}\end{array}$ & n.a. & 20 Aug. 1969 & $1 /-$ & 47 & $\begin{array}{l}\text { SMNS } \\
14366\end{array}$ \\
\hline & GR5 & Greece & $\begin{array}{l}\text { Porto Zografou, } 24 \mathrm{~km} \text { southeast of } \\
\text { Nikiti, east coast, Sithonia, Chalkidiki }\end{array}$ & $\begin{array}{ll}40^{\circ} 06^{\prime} \mathrm{N} \\
\mathrm{i} \quad 23^{\circ} 54^{\prime} \mathrm{E}\end{array}$ & n.a. & 18 Aug. 1994 & $2 /-$ & $59-62$ & $\begin{array}{r}\text { SMNS } \\
15737\end{array}$ \\
\hline & GR6 & Greece & Palaiokastrizza, Korfu/Corfu Island & $\begin{array}{l}39^{\circ} 43^{\prime} \mathrm{N} \\
19^{\circ} 38^{\prime} \mathrm{E}\end{array}$ & n.a. & 11 July 1977 & $2 /-$ & $57-64$ & $\begin{array}{l}\text { SMNS } \\
8395\end{array}$ \\
\hline & MO1 1 & Montenegro & $\begin{array}{l}\text { Bay north of Budva, } \\
\text { right side of river mouth }\end{array}$ & $\begin{array}{l}42^{\circ} 16^{\prime} 30^{\prime}, \mathrm{N} \\
18^{\circ} 50^{\prime} 30^{\prime} \mathrm{E}\end{array}$ & n.a. & 7 May 1977 & $1 /-$ & 55 & $\begin{array}{l}\text { SMNS } \\
13609\end{array}$ \\
\hline & MO2 & Montenegro & Bay of Kotor, at Bijela & $\begin{array}{l}42^{\circ} 27^{\prime} \mathrm{N} \\
18^{\circ} 41^{\prime} \mathrm{E}\end{array}$ & n.a. & 20 May 1969 & $1 /-$ & 45 & $\begin{array}{l}\text { SMNS } \\
14327\end{array}$ \\
\hline & CR1 & Croatia & $\begin{array}{l}\text { Lokrum Island, } \\
\text { west shore, Dubrovnik }\end{array}$ & $\begin{array}{l}42^{\circ} 37^{\prime} 36^{\prime \prime}, \mathrm{N} \\
18^{\circ} 07^{\prime} 07^{\prime \prime} \mathrm{E}\end{array}$ & n.a. & 21 Sep. 1987 & $6 / 0$ & $32-53$ & $\begin{array}{l}\text { SMNS } \\
8391\end{array}$ \\
\hline & CR2a & Croatia & $\begin{array}{l}\text { Tatinica, northwest coast, } \\
\text { Mljet Island }\end{array}$ & $\begin{array}{l}42^{\circ} 46^{\prime} 34^{\prime \prime}, \mathrm{N} \\
17^{\circ} 27^{\prime} 59^{\prime \prime} \mathrm{E}\end{array}$ & n.a. & 18 Sep. 1987 & $1 / 0$ & 46 & $\begin{array}{l}\text { SMNS } \\
8400\end{array}$ \\
\hline
\end{tabular}


TABLE 1 (cont.). - Specimens of the two Tripterygion species collected at different localities of the Mediterranean and Atlantic adjacent waters. The number of individuals used for morphological $(\mathrm{Nm})$ and molecular $(\mathrm{Ng})$ analyses, for each locality, are detailed.

\begin{tabular}{|c|c|c|c|c|c|c|c|c|}
\hline Map code & Country & Locality & $\begin{array}{l}\text { Latitude/ } \\
\text { Longitude }\end{array}$ & $\begin{array}{l}\text { Depth } \\
(\mathrm{m})\end{array}$ & $\begin{array}{l}\text { Collection } \\
\text { date }\end{array}$ & $\begin{array}{l}\mathrm{Nm} / \\
\mathrm{Ng}\end{array}$ & $\begin{array}{l}\text { LT range } \\
(\mathrm{mm})\end{array}$ & $\begin{array}{l}\text { Catalogue } \\
\text { number }\end{array}$ \\
\hline CR2b & Croatia & southwest coast, Mljet Island & $\begin{array}{l}42^{\circ} 45,52,{ }^{\prime} \mathrm{N} \\
17^{\circ} 21,51, ' \mathrm{E}\end{array}$ & n.a. & 17 Sep. 1987 & $3 / 0$ & $54-68$ & $\begin{array}{l}\text { SMNS } \\
8404\end{array}$ \\
\hline CR3 & Croatia & $\begin{array}{l}\text { Sestrice Island, } \\
\text { near Orebic, Peljesac }\end{array}$ & $\begin{array}{l}42^{\circ} 56^{\prime} \mathrm{N} \\
17^{\circ} 08{ }^{\prime} \mathrm{E}\end{array}$ & n.a. & 9 Aug. 1963 & $2 /-$ & $62-63$ & $\begin{array}{l}\text { SMNS } \\
14325\end{array}$ \\
\hline CR4 & Croatia & Orebic, Peljesac & $\begin{array}{l}42^{\circ} 56^{\prime} \mathrm{N} \\
17^{\circ} 08^{\prime} \mathrm{E}\end{array}$ & n.a. & 7 Aug. 1963 & $1 / 0$ & 52 & $\begin{array}{l}\text { SMNS } \\
14370\end{array}$ \\
\hline CR5 & Croatia & $\begin{array}{c}\text { Gojak Island, } 12 \mathrm{~km} \\
\text { southeast of Kardeljevo }\end{array}$ & $\begin{array}{l}42^{\circ} 57^{\prime} \mathrm{N} \\
17^{\circ} 27^{\prime} \mathrm{E}\end{array}$ & n.a. & 20 Aug. 1963 & $5 / 0$ & $42-47$ & $\begin{array}{l}\text { SMNS } \\
14362\end{array}$ \\
\hline CR6 & Croatia & $\begin{array}{l}\text { Badija Islet, beach at north } \\
\text { coast of islet, east of Korçula city, } \\
\text { Korcula Island }\end{array}$ & $\begin{array}{l}42^{\circ} 57^{\prime} 28^{\prime}, \mathrm{N} \\
17^{\circ} 09^{\prime} 43^{\prime}, \mathrm{E}\end{array}$ & n.a. & 9 Sep. 1987 & $4 / 0$ & $41-51$ & $\begin{array}{l}\text { SMNS } \\
8399\end{array}$ \\
\hline CR7 & Croatia & Podaca, $12 \mathrm{~km}$ northwest of Ploce & $\begin{array}{l}43^{\circ} 09^{\prime} \mathrm{N} \\
17^{\circ} 15^{\prime} \mathrm{E}\end{array}$ & n.a. & 6 Aug. 1963 & $1 / 0$ & 51 & $\begin{array}{l}\text { SMNS } \\
14368\end{array}$ \\
\hline CR8a & Croatia & Hvar City, Hvar Island & $\begin{array}{l}43^{\circ} 10^{\prime} 09^{\prime}, \mathrm{N} \\
16^{\circ} 26^{\prime} 31^{\prime}, \mathrm{E}\end{array}$ & n.a. & 26 Sep. 1987 & $6 / 0$ & $39-54$ & $\begin{array}{l}\text { SMNS } \\
8401\end{array}$ \\
\hline CR8b & Croatia & Jerolim Islet, near Hvar, & $\begin{array}{l}43^{\circ} 09,28, ' \mathrm{~N} \\
16^{\circ} 233^{\prime},{ }^{\prime} \mathrm{E}\end{array}$ & n.a. & 25 Sep. 1987 & $4 / 0$ & $27-49$ & $\begin{array}{l}\text { SMNS } \\
8393\end{array}$ \\
\hline CR8c & Croatia & Lesina/Hvar City, Hvar Island & $\begin{array}{l}43^{\circ} 10^{\prime} \mathrm{N} \\
16^{\circ} 27^{\prime} \mathrm{E}\end{array}$ & n.a. & $\begin{array}{l}\text { cat. entry } \\
\text { June } 1854\end{array}$ & $2 / 0$ & $53-57$ & $\begin{array}{l}\text { SMNS } \\
420\end{array}$ \\
\hline CR9 & Croatia & Bay of Rogoznica, at Rogoznica & $\begin{array}{l}43^{\circ} 311^{\prime} 10^{\prime \prime}, \mathrm{N} \\
15^{\circ} 59^{\prime} 00^{\prime}, \mathrm{E}\end{array}$ & n.a. & 8 Sep. 1987 & $1 / 0$ & 22 & $\begin{array}{l}\text { SMNS } \\
25179\end{array}$ \\
\hline CR10a & Croatia & Biograd & $\begin{array}{l}43^{\circ} 55^{\prime} \mathrm{N} \\
15^{\circ} 23^{\prime} \mathrm{E}\end{array}$ & n.a. & 7 Aug. 1959 & $2 / 0$ & $38-45$ & $\begin{array}{l}\text { SMNS } \\
13365\end{array}$ \\
\hline CR10b & Croatia & Biograd & $\begin{array}{l}43^{\circ} 55^{\prime} \mathrm{N} \\
15^{\circ} 23^{\prime} \mathrm{E}\end{array}$ & n.a. & 4-6 Aug. 1961 & $5 / 0$ & $38-57$ & $\begin{array}{l}\text { SMNS } \\
13364\end{array}$ \\
\hline CR11 & Croatia & $\begin{array}{l}\text { Karlobag, coast at } \\
\text { northern entrance into town }\end{array}$ & $\begin{array}{l}44^{\circ} 32^{\prime} \mathrm{N} \\
15^{\circ} 04^{\prime} \mathrm{E}\end{array}$ & n.a. & 5 Aug. 1963 & $2 / 0$ & $50-55$ & $\begin{array}{l}\text { SMNS } \\
13605\end{array}$ \\
\hline CR12 & Croatia & $\begin{array}{l}\text { Gavza Bay, } 3 \mathrm{~km} \text { northwest } \\
\text { of Cres City, Cres Island }\end{array}$ & $\begin{array}{l}44^{\circ} 59^{\prime} 24^{\prime \prime} \mathrm{N} \\
14^{\circ} 23^{\prime} 24^{\prime}, \mathrm{E}\end{array}$ & n.a. & 2 May 1989 & $2 / 0$ & $61-64$ & $\begin{array}{l}\text { SMNS } \\
8664\end{array}$ \\
\hline CR13a & Croatia & Osor, Cres Island & $\begin{array}{l}44^{\circ} 42^{\prime} \mathrm{N} \\
14^{\circ} 23^{\prime} \mathrm{E}\end{array}$ & 0.7 & 12 Sep. 1989 & $2 / 0$ & $36-43$ & $\begin{array}{c}\text { SMNS } \\
9428\end{array}$ \\
\hline CR13b & Croatia & Osor, Cres Island & $\begin{array}{l}44^{\circ} 42^{\prime} \mathrm{N} \\
14^{\circ} 23^{\prime} \mathrm{E}\end{array}$ & n.a. & Sep. 1989 & $3 / 0$ & $35-61$ & $\begin{array}{l}\text { SMNS } \\
9425\end{array}$ \\
\hline CR13c & Croatia & Osor, Cres Island & $\begin{array}{l}44^{\circ} 42^{\prime} \mathrm{N} \\
14^{\circ} 23^{\prime} \mathrm{E}\end{array}$ & 0.2 & 27 Sep. 1989 & $3 / 0$ & $32-55$ & $\begin{array}{l}\text { SMNS } \\
9423\end{array}$ \\
\hline CR13d & Croatia & Osor, Cres Island & $\begin{array}{l}44^{\circ} 42^{\prime} \mathrm{N} \\
14^{\circ} 23^{\prime} \mathrm{E}\end{array}$ & n.a. & Sep. 1990 & $4 / 0$ & $35-63$ & $\begin{array}{l}\text { SMNS } \\
11236\end{array}$ \\
\hline CR14 & Croatia & Cres City, Cres Island & $\begin{array}{l}44^{\circ} 57^{\prime} 24^{\prime \prime}, \mathrm{N} \\
14^{\circ} 24^{\prime} 21^{\prime}, \mathrm{E}\end{array}$ & n.a. & 3 May 1989 & $3 / 0$ & $43-63$ & $\begin{array}{l}\text { SMNS } \\
9214\end{array}$ \\
\hline CR15a & Croatia & $\begin{array}{l}\text { Zlatne Stijene, } 5 \mathrm{~km} \\
\text { south of Pula, Istria }\end{array}$ & $\begin{array}{l}44^{\circ} 50,30, ' \mathrm{~N} \\
13^{\circ} 50^{\prime} 30^{\prime \prime} \mathrm{E}\end{array}$ & n.a. & 10 June 1978 & $2 / 0$ & $\begin{array}{l}60-63 \\
8410\end{array}$ & SMNS \\
\hline CR15b & Croatia & $\begin{array}{l}\text { Zlatne Stijene, } 5 \mathrm{~km} \\
\text { south of Pula, Istria }\end{array}$ & $\begin{array}{l}44^{\circ} 500^{\prime} 30, ' \mathrm{~N} \\
13^{\circ} 50^{\prime} 30, ' \mathrm{E}\end{array}$ & n.a. & 9 June 1978 & $5 / 0$ & $47-60$ & $\begin{array}{l}\text { SMNS } \\
8396\end{array}$ \\
\hline IT1 & Italy & Lecce, Harbour & $\begin{array}{l}40^{\circ} 13^{\prime} 45^{\prime \prime}, \mathrm{N} \\
18^{\circ} 06^{\prime} 30^{\prime}, \mathrm{E}\end{array}$ & $0-2$ & 5 June 2004 & $5 / 4$ & $36-59$ & IIPB \\
\hline IT2 & Italy & Sicily Is., Messina Harbour & $\begin{array}{l}38^{\circ} 11^{\prime} \mathrm{N} \\
15^{\circ} 33^{\prime} \mathrm{E}\end{array}$ & $0-2$ & 4 Jan. 2006 & $5 / 5$ & $49-63$ & IIPB \\
\hline FR1 & France & Corsica Is., Ile Rousse & $\begin{array}{l}42^{\circ} 37^{\prime} 39^{\prime \prime} \mathrm{N} \\
8^{\circ} 55^{\prime} 37^{\prime}, \mathrm{E}\end{array}$ & $0-2$ & 24 Aug. 2004 & $2 / 2$ & $28-31$ & $\mathrm{MNCN}$ \\
\hline FR2 & France & Nice, Harbour & $\begin{array}{l}43^{\circ} 25^{\prime} 16^{\prime \prime} \mathrm{N} \\
7^{\circ} 08^{\prime} 24, ' \mathrm{E}\end{array}$ & $0-2$ & 13 Mar. 2005 & $2 / 2$ & $49-58$ & $\mathrm{MNCN}$ \\
\hline SP1 & Spain & Port de la Selva, Harbour & $\begin{array}{l}42^{\circ} 42^{\prime} 38^{\prime \prime} \mathrm{N} \\
3^{\circ} 19^{\prime} 50^{\prime \prime} \mathrm{E}\end{array}$ & $0-2$ & 12 Aug. 2004 & $12 / 1$ & $43-63$ & IIPB \\
\hline SP2 & Spain & Blanes, St.Francesc Bay & $\begin{array}{l}41^{\circ} 400^{\prime} 09^{\prime \prime} \mathrm{N} \\
2^{\circ} 48^{\prime} 155^{\prime} \mathrm{E}\end{array}$ & $0-2$ & $30 \mathrm{Jul} .2002$ & $9 / 2$ & $45-65$ & MNCN \\
\hline SP3 & Spain & Tarragona, Altafulla & $\begin{array}{l}41^{\circ} 05^{\prime} 35^{\prime}, \mathrm{N} \\
1^{\circ} 13^{\prime} 45^{\prime}, \mathrm{E}\end{array}$ & $0-2$ & 20 Jul. 2003 & $19 / 3$ & $51-62$ & IIPB \\
\hline SP4 & Spain & Columbretes Is., La Foradada & $\begin{array}{l}39^{\circ} 533^{\prime} 50, ' \mathrm{~N} \\
0^{\circ} 41^{\prime} 155^{\prime} \mathrm{E}\end{array}$ & $0-2$ & 4 Aug. 2002 & $4 / 3$ & $38-47$ & $\mathrm{MNCN}$ \\
\hline MA1a & Malta & $\begin{array}{c}\text { Cirkewwa/Paradise Bay, southwest } \\
\text { corner of bay, northwest coast, } \\
\text { Malta Island }\end{array}$ & $\begin{array}{l}36^{\circ} 58^{\prime} 56^{\prime \prime} \mathrm{N} \\
14^{\circ} 19^{\prime} 56^{\prime}, \mathrm{E}\end{array}$ & n.a. & 10 Apr. 1974 & $1 /-$ & 58 & $\begin{array}{l}\text { SMNS } \\
13045\end{array}$ \\
\hline MA1b & Malta & $\begin{array}{c}\text { Cirkewwa/Paradise Bay, southwest } \\
\text { corner of bay, northwest coast, } \\
\text { Malta Island }\end{array}$ & $\begin{array}{l}36^{\circ} 58^{\prime} 56^{\prime \prime} \mathrm{N} \\
14^{\circ} 19^{\prime} 56^{\prime} \mathrm{E}\end{array}$ & $0-1.5$ & 10 Aug. 2005 & $7 /-$ & $30-44$ & $\begin{array}{l}\text { SMNS } \\
24888\end{array}$ \\
\hline MA1c & Malta & $\begin{array}{c}\text { Cirkewwa/Paradise Bay, southwest } \\
\text { corner of bay, northwest coast, } \\
\text { Malta Island }\end{array}$ & $\begin{array}{l}36^{\circ} 58^{\prime} 56^{\prime \prime}, \mathrm{N} \\
14^{\circ} 19^{\prime} 56^{\prime} \mathrm{E}\end{array}$ & $0-1.2$ & 12 Aug. 2005 & 4/- & $29-47$ & $\begin{array}{l}\text { SMNS } \\
24911\end{array}$ \\
\hline MA1d & Malta & $\begin{array}{c}\text { Cirkewwa/Paradise Bay, southwest } \\
\text { corner of bay, northwest coast, } \\
\text { Malta Island }\end{array}$ & $\begin{array}{l}36^{\circ} 58^{\prime} 56^{\prime}, \mathrm{N} \\
14^{\circ} 19^{\prime} 56^{\prime}, \mathrm{E}\end{array}$ & $0-1.5$ & 11 Aug. 2005 & $1 /-$ & 25 & $\begin{array}{l}\text { SMNS } \\
24899\end{array}$ \\
\hline
\end{tabular}


TABle 1 (cont.). - Specimens of the two Tripterygion species collected at different localities of the Mediterranean and Atlantic adjacent waters. The number of individuals used for morphological $(\mathrm{Nm})$ and molecular $(\mathrm{Ng})$ analyses, for each locality, are detailed.

\begin{tabular}{|c|c|c|c|c|c|c|c|c|c|}
\hline & Map code & Country & Locality & $\begin{array}{l}\text { Latitude/ } \\
\text { Longitude }\end{array}$ & $\begin{array}{l}\text { Depth } \\
\text { (m) }\end{array}$ & $\begin{array}{l}\text { Collection } \\
\text { date }\end{array}$ & $\begin{array}{l}\mathrm{Nm} / \\
\mathrm{Ng}\end{array}$ & $\begin{array}{l}\text { LT range } \\
(\mathrm{mm})\end{array}$ & $\begin{array}{c}\text { Catalogue } \\
\text { number }\end{array}$ \\
\hline $\begin{array}{l}\text { Tripterygion } \\
\text { tartessicum }\end{array}$ & SP5 & Spain & Menorca Is., Fornells Bay & $\begin{array}{l}40^{\circ} 04^{\prime} 23^{\prime \prime} \mathrm{N} \\
4^{\circ} 08^{\prime} 31^{\prime}, \mathrm{E}\end{array}$ & $0-2$ & 5 Jul. 2002 & $2 / 2$ & $50-60$ & IIPB \\
\hline PARATYPES & SP6 & Spain & Formentera Is., Punta Prima & $\begin{array}{c}38^{\circ} 44^{\prime} \mathrm{N} \\
1^{\circ} 25^{\prime} 14^{\prime}, \mathrm{E}\end{array}$ & $0-2$ & 7 May 2003 & $2 / 2$ & $59-60$ & MNCN \\
\hline PARATYPES & SP7 & Spain & Cabo La Nao, Dènia - Les Rotes & $\begin{array}{l}38^{\circ} 51^{\prime} \mathrm{N} \\
0^{\circ} 07^{\prime} \mathrm{E}\end{array}$ & $0-2$ & 5 May 2005 & $4 / 4$ & $48-63$ & IIPB \\
\hline PARATYPES & SP8 & Spain & Cabo de Palos, Phare & $\begin{array}{l}37^{\circ} 37^{\prime} 57^{\prime \prime} \mathrm{N} \\
0^{\circ} 41^{\prime} 56^{\prime}, \mathrm{W}\end{array}$ & $0-2$ & 30 Oct. 2002 & $12 / 2$ & $41-60$ & IIPB \\
\hline PARATYPES & SP9 & Spain & Cabo de Gata, Aguamarga, Almería & $\begin{array}{l}36^{\circ} 59^{\prime} 43^{\prime \prime}, \mathrm{N} \\
1^{\circ} 53^{\prime} 41^{\prime \prime} \mathrm{W}\end{array}$ & $0-2$ & 26 Oct. 2002 & $16 / 1$ & $38-67$ & MNCN \\
\hline PARATYPES & SP10 & Spain & Tarifa, Las Palomas Is. & $\begin{array}{l}36^{\circ} 00^{\prime} 15^{\prime \prime} \mathrm{N} \\
5^{\circ} 36^{\prime} 30^{\prime}, \mathrm{W}\end{array}$ & $0-2$ & 20 Oct 2003 & $2 / 2$ & $42-65$ & MNCN \\
\hline PARATYPES & SP11 & $\begin{array}{l}\text { Spain } \\
\text { (Africa) }\end{array}$ & Ceuta, Harbour & $\begin{array}{l}35^{\circ} 53^{\prime} \mathrm{N} \\
5^{\circ} 18^{\prime} \mathrm{W}\end{array}$ & $0-2$ & 12 Feb. 2005 & $3 / 3$ & $28-53$ & MNCN \\
\hline HOLOTYPE & SP12 & Spain & Cádiz, Puercas Phare & $\begin{array}{l}36^{\circ} 18^{\prime} \mathrm{N} \\
6^{\circ} 12^{\prime} \mathrm{W}\end{array}$ & $0-2$ & 22 Oct. 2003 & $1 /$ & 67 & $\begin{array}{l}\text { IIPB } \\
15 / 2005\end{array}$ \\
\hline PARATYPES & SP12 & Spain & Cádiz, Puercas Phare & $\begin{array}{l}36^{\circ} 18^{\prime} \mathrm{N} \\
6^{\circ} 12^{\prime} \mathrm{W}\end{array}$ & $0-2$ & 22 Oct. 2003 & $12 / 2$ & $31-52$ & $\begin{array}{l}\text { IIPB } \\
15 / 2005\end{array}$ \\
\hline PARATYPES & SP13a & Spain & $\begin{array}{c}1 \mathrm{~km} \mathrm{SW} \text { of Punta de la Chullera, } \\
\text { at Torreguadiaro (150 m northeast), } \\
\text { Cádiz, Andalucía }\end{array}$ & $\begin{array}{l}36^{\circ} 18^{\prime} 23^{\prime \prime} \mathrm{N} \\
5^{\circ} 15^{\prime} 39^{\prime \prime} \mathrm{W}\end{array}$ & $0-1.5$ & 14 Aug. 2004 & $2 / 0$ & $72-73$ & $\begin{array}{l}\text { SMNS } \\
24307\end{array}$ \\
\hline PARATYPES & SP13b & Spain & $\begin{array}{l}1 \mathrm{~km} \mathrm{SW} \text { of Punta de la Chullera, } \\
\text { at Torreguadiaro ( } 150 \mathrm{~m} \text { northeast), } \\
\text { Province Cádiz, Andalucía }\end{array}$ & $\begin{array}{l}36^{\circ} 18^{\prime} 23^{\prime \prime} \mathrm{N} \\
5^{\circ} 15^{\prime} 39^{\prime \prime} \mathrm{W}\end{array}$ & $0-1.5$ & 19 Aug. 2004 & $2 / 0$ & $63-77$ & $\begin{array}{l}\text { SMNS } \\
24327\end{array}$ \\
\hline PARATYPES & MC & Morocco & Plage David & n.a. & n.a. & June 1985 & $11 /-$ & $42-69$ & $\begin{array}{l}\text { SMNS } \\
13516\end{array}$ \\
\hline PARATYPE & TU1a & Tunisia & $\begin{array}{l}\text { Rocky cape, } 4 \mathrm{~km} \text { east of Tabarca, } \\
66 \mathrm{~km} \text { east of Bone/Annaba (Algeria) }\end{array}$ & $\begin{array}{l}36^{\circ} 57^{\prime} 33^{\prime \prime} \mathrm{N} \\
8^{\circ} 47^{\prime} 54^{\prime \prime} \mathrm{E}\end{array}$ & $0.1-2$ & 2 June 1998 & $1 /-$ & 58 & $\begin{array}{l}\text { SMNS } \\
20366\end{array}$ \\
\hline PARATYPE & TU1b & Tunisia & $\begin{array}{l}\text { Rocky cape, } 4 \mathrm{~km} \text { east of Tabarca, } \\
66 \mathrm{~km} \text { east of Bone/Annaba (Algeria) }\end{array}$ & $\begin{array}{l}36^{\circ} 57^{\prime} 33^{\prime \prime} \mathrm{N} \\
8^{\circ} 47^{\prime} 54^{\prime \prime} \mathrm{E}\end{array}$ & $0-1.8$ & 27 May 1998 & $1 /-$ & 46 & $\begin{array}{l}\text { SMNS } \\
20356\end{array}$ \\
\hline PARATYPES & TU1c & Tunisia & $\begin{array}{l}\text { Rocky cape, } 4 \mathrm{~km} \text { east of Tabarca, } \\
66 \mathrm{~km} \text { east of Bone/Annaba (Algeria) }\end{array}$ & $\begin{array}{l}36^{\circ} 57^{\prime} 33^{\prime \prime} \mathrm{N} \\
8^{\circ} 47^{\prime} 54^{\prime \prime} \mathrm{E}\end{array}$ & $0-0.6$ & 23 May 1998 & $2 /-$ & $52-61$ & $\begin{array}{l}\text { SMNS } \\
20342\end{array}$ \\
\hline
\end{tabular}

(0): no amplifications were done, (-): amplifications were done but they did not succeed, (n.a.): no available data. The holotype and paratypes are labelled; the catalogue number for each individual is shown. (IIPB): Instituto de Ciencias del Mar de Barcelona, (SMNS): Staatliches Museum fuer Naturkunde Stuttgart. The first two letters in the map code identify each country, the number identifies the locality and the lower case letter identifies different collection dates.

resulting in 15000 trees. The first 1500 trees were eliminated since they did not reach the stationarity of the likelihood values and the rest were used to construct the consensus tree and obtain the posterior probabilities of the branches.

\section{SYSTEMATIC ACCOUNT}

Tripterygion tartessicum $\mathrm{n}$. $\mathrm{sp}$.

(Figs. 2 and 3a)

Etymology. The name tartessicum referred to the old Spanish culture (Tartessos, at least dating from $1000 \mathrm{BC}$ ) located on the south coast of the Iberian peninsula (in modern Andalusia, Spain), where the new species is partially distributed.

Morphological description. Body elongate and compressed, greatest height at base of anal fin, being about one-sixth total length. Scales ctenoid, covering entire body except base of pectoral fin and ventral abdominal region back to vent. Lateral line having two sections: anterior section with 20 (19-22) pored scales, posterior section with 22 (21-24) notched scales, having 42 (40-46) in total. Upper, anterior, section commencing at upper angle of opercular opening, slightly curving up over pectoral fin base and running parallel to dorsal profile to point below last 1-3 rays of second dorsal fin; canal running across exposed width of each scale. Lower, posterior, section commencing below, and in front of last scale or two of upper section, running along the mid-line of tail to caudal fin base; each scale with shallow notch in free-edge tip.

Three dorsal fins with III + XVI + 13 (III + XVIXVIII + 12-13) rays. First dorsal fin lower than second and second higher than third. First just above pre-operculum, rays being of equal height. Second separated by short interspaces, origin slightly behind 


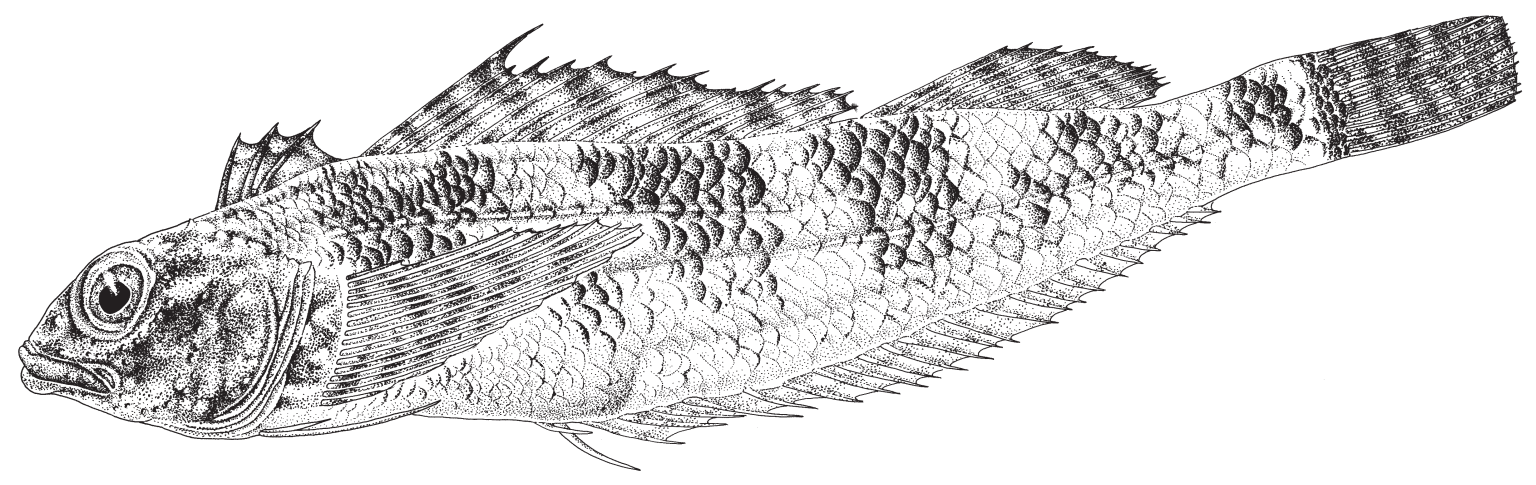

FIG. 2. - Tripterygion tartessicum Holotype, IIPB 15/2005, male, 67 mm TL, from SP12.

base of the pectoral fin; first ray longest, in mature males being nearly twice as long as rays in middle region, with distal half not united by membrane with following ray. Base of third fin about 0.6 length of second dorsal fin base.

Caudal fin truncate, with $\mathrm{X}$ (IX-X) principal branched rays, and 2 (2-3) procurrent lower and upper.

Anal fin elongate and of uniform height, with II +23 (II + 22-24) rays. Anteriorly, 2 weak, slender, unsegmented rays, first shorter than second, which is slightly longer than the first segmented ray; succeeding rays united by membrane and decreasing in length posteriorly.

Pectorals long and broad, slightly overreaching mid-length of second dorsal fin and base of anal fin; with 16 (15-16) rays, upper three rays short and simple, remainder branched; ninth ray, counted from upper edge, longer than others.

Pelvic fins with one short spine and two slender and segmented rays; longest ray reaching midlength of pectoral fin.

Head broad, scale less, profile acute, lips prominent. Head length $0.19(0.16-0.22)$ times total length (TL). Orbit large, almost circular, diameter 0.32 (0.28-0.51) times head length, upper edge forming ridge along upper head profile. Pre-orbital and predorsal lengths 0.05 and 0.14 times total length, respectively ( 0.06 and 0.18 ). Interorbital region concave. Mouth nearly horizontal, maxilla extending to level of front of pupil. Gill membrane continuous across throat. Teeth conical, in band in upper and lower jaws. Anterior nostril tubular, posterior nostril close to orbit edge. Cephalic canal pores as illustrated in Figure 3a, with preopercular-dentary series complete. The mandibular pore formula (Fricke, 1997) was $3+2+3(3-4+2+3-4)$, basically depending on the fish TL, suggesting that an increase in length could be associated with the appearance of a new pore in both dentaries. However, no significant relationship was found between this formula and TL, or between the two species. The interorbital series 2 (24) opened singly from the upper interorbital region to the upper lip. The preopercular series opened singly along the lower side of the preopercular canal, opening in pairs on the posterior pre-opercular edge. The nasal and suborbital canals usually opened in pairs, running along the lower and the posterior margins of the orbit; nasal pores 3 (1 to 3 ) placed in front of the anterior border of the eye;
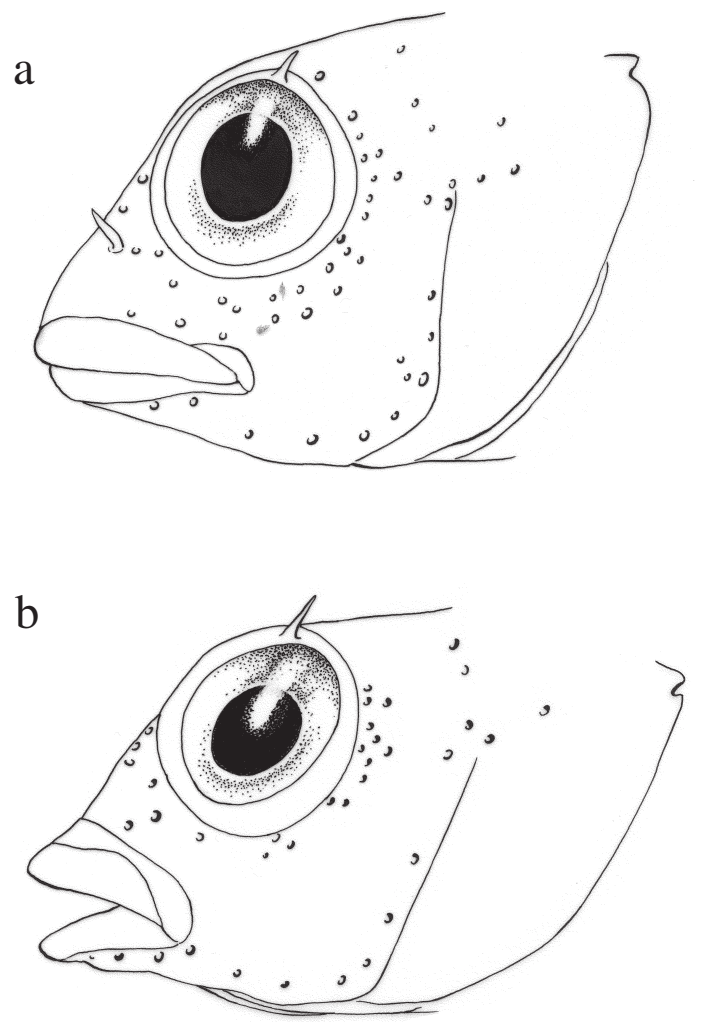

FIG. 3. - Variation in the cephalic pore system between T.tartessicum (a, female $55 \mathrm{~mm}$ from SP9) and T. tripteronotus (b, female $58 \mathrm{~mm}$ from SP1). 
outer branch of suborbital pores ending as a cluster of pores in the postorbital region. Some nuchal pores running from the upper part of the operculum across the nape to the opposite side. Postocular canal with single pores (Fig. 3a).

Colouration in life. Mature males during reproduction period (March-August): black head, extending posteriorly to first dorsal, laterally to operculum edge, and ventrally including branchiostegal membranes across throat, base of pectoral fins and pelvic rays. Red body. Caudal fin with 4 red bars (dark brown in preserved specimens). First dorsal fin rays and membrane heavily pigmented. Anal fin with dusky marks, membrane hyaline. Pectoral fins hyaline, median rays with dusky margins on basal third. The rest of the year their colouration is as females or immature males (sneakers).

Females and immature males (sneakers): head and body light brown with dark bars across flanks, last bar not forming extension onto base of caudal fin. First dorsal fin heavily pigmented both on rays and membrane, second and third dorsal fins with brownish bars. Caudal fin with 4 distinct brownish bars.

Habitat. The new species inhabits similar habitats to T. tripteronotus: shallow rocky shores to $6 \mathrm{~m}$, preferably between 0 and $3 \mathrm{~m}$; in light-exposed and shady biotopes dominated by algal communities (e.g. Corallina elongata, Cladophora spp., Litophyllum spp., Enteromorpha spp.). Nests are usually situated in sciaphyl habitats dominated by steep rocky zones, without arborescent algae.

\section{Comparison between $T$. tripteronotus and T. tartessicum}

\section{Background}

Tripterygion tripteronotus was described by Risso (1810) as Blennius tripteronotus, from specimens collected in Nice (France; FR2). Unfortunately the types seem to be lost. Subsequently, the species was named as T. nasus (Risso, 1826) from material collected in Nice (France; FR2), T. melaenocephalus (Cocco, 1829) from specimens collected in Messina (Italy; IT2), and Tripterygium nikolskii (Maksimov, 1909) from the Crimea (Ukraine, Black Sea). These names were considered as junior synonyms of $T$. tripteronotus (see Hureau and Monod, 1973; Zander, 1986). Zander and Heymer $(1970,1976)$ mentioned

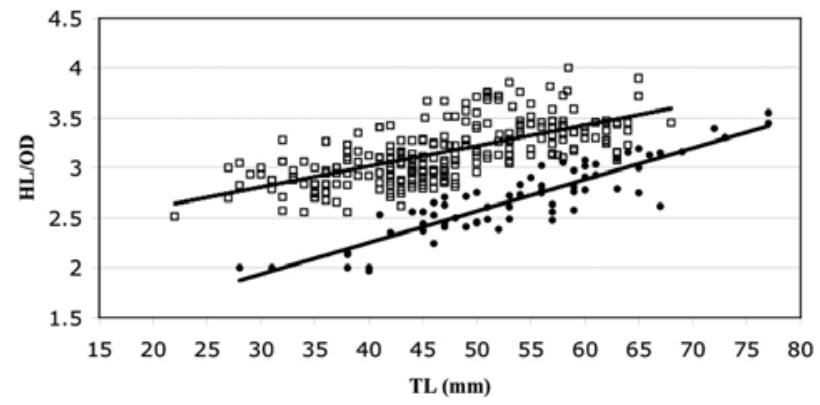

FIG. 4. - Plotted relationship between Total Length (TL, mm) and Head Length (HL, mm) / Orbital Diameter (OD, mm) for all measured individuals from the two species. (๑): T. tripteronotus, $(\square): T$. tartessicum. Regression equations are HL/OD $=0.0207 \mathrm{TL}+2.1884$ $\left(\mathrm{R}^{2}=0.42\right)$ and $\mathrm{HL} / \mathrm{OD}=0.0315 \mathrm{TL}+0.991\left(\mathrm{R}^{2}=0.80\right)$ respectively.

some slight morphological differences between specimens from different localities (NW Mediterranean and Mediterranean coasts of Morocco and Israel), although they were considered as intraspecific variations.

\section{Morphological data}

The morphological comparison of the present material of $T$. tartessicum with specimens of $T$. tripteronotus from different localities of the Mediterranean and adjacent waters showed that there are only slight differences between the two species. They can be differentiated by a morphometric measurement: the orbital diameter (OD) is significantly longer in the new species (mean ratio head length/orbital diameter $=2.69 \pm 0.36)$ than in $T$. tripteronotus (3.16 \pm 0.29 ; Mann-Whitney U-test, $\mathrm{p}<0.05)$. When HL/OD was represented in front of TL, two well-differentiated and almost non-overlapping groups were found, corresponding to both species (Fig. 4). In order to assure this differentiation, a multivariate analysis of covariance (MANCOVA) was implemented using TL as the covariate and HL/OD as the dependent variable. The results showed a highly significant differentiation between the two groups $(F=415.72, p<0.001)$.

The first ray of the second dorsal fin of the mature males has the distal half not united by a membrane with the following ray in T. tripteronotus, whereas the first two rays can be united by a membrane from their respective tips in $T$. tartessicum. Additionally, the caudal fin usually has four red or brownish bars (black in preserved specimens) in the new species, whereas these bars are usually not distinct in T. tripteronotus. These two differences are similar to the ones described by Zander and Heymer 


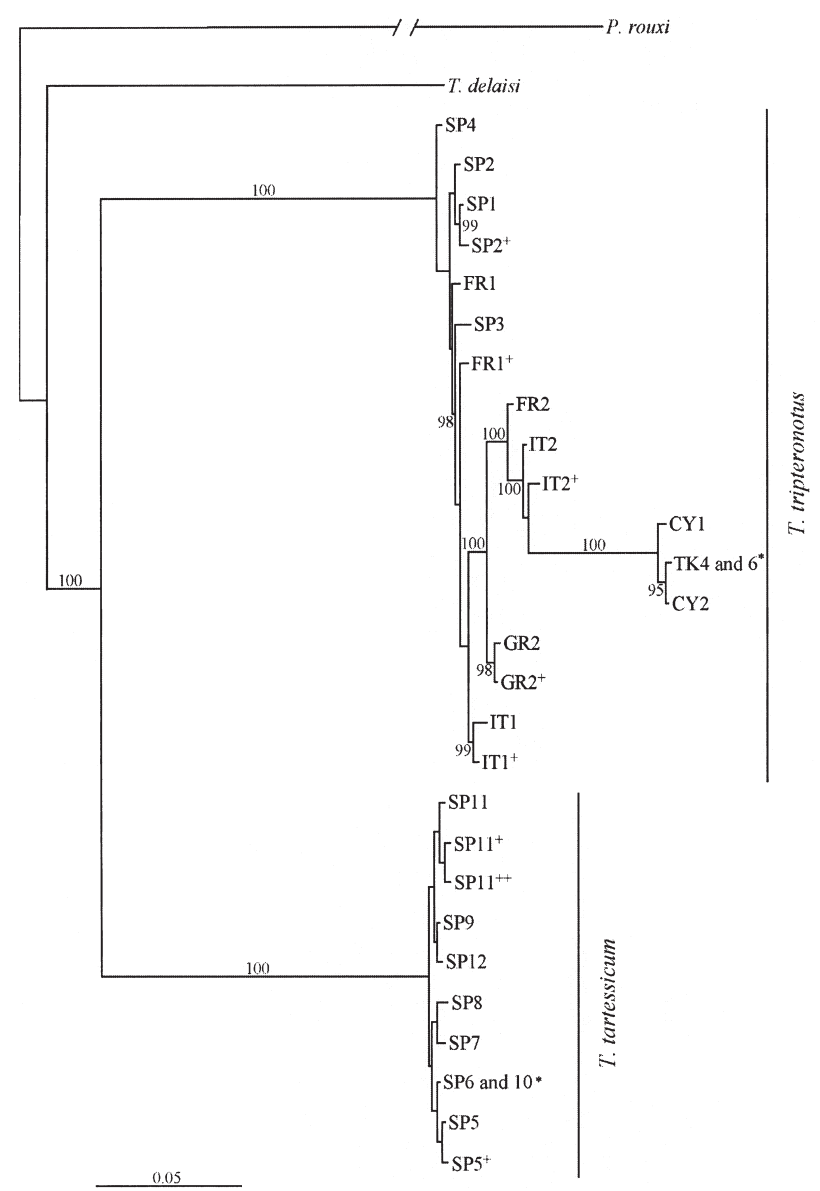

FIG. 5. - Haplotype tree inferred from Bayesian Inference for $T$. tartessicum and T. tripteronotus species using all genes together. Only probabilities above $95 \%$ are shown; $\left({ }^{+}\right.$and $\left.{ }^{++}\right)$: different haplotypes found in the same locality; $\left(^{*}\right)$ : the same haplotype found in different localities. See Table 1 for locality abbreviations and further details or Figure 1 for a quick geographical location.

(1976), although they should be considered with caution since they were not always observable in all the individuals collected.

We also observed that the mating season seems to start slightly later in the new species. In fact, all mature males of $T$. tripteronotus are active on the Catalan coast (NE Spain) in early May, whereas most mature males of the new species are not active at this time on the coasts of Murcia and Almeria (SE Spain).

\section{Molecular data}

We analysed a total of $1732 \mathrm{bp}$ for all genes combined in 55 individuals (18 T. tartessicum and $37 \mathrm{~T}$. tripteronontus). A total of 10 haplotypes were found for $T$. tartessicum, whereas 17 were shown for $T$. tripteronotus (Fig. 5). Generally, all individuals from one locality shared the same haplotype, and
TK4-TK6 and SP6-SP10 also shared the same haplotype. However, at some localities (SP2, FR1, IT1, IT2, GR2, SP5 and SP11) more than one haplotype was found. For each of the four mitochondrial genes the sequence obtained was of 419 bp for $12 \mathrm{~S}$ rRNA, 699 bp for $16 \mathrm{~S}$ rRNA, 73 bp for tRNA-valine and $541 \mathrm{bp}$ for Cytochrome Oxidase I. All genes used showed a similar percentage of parsimony informative sites (chi-square $=7.57 \mathrm{p}=0.36$ ) ranging from 2.74 to $10.35 \%$, but only the RNA genes had similar variable sites (chi-square $=7.52 \mathrm{p}=0.18$ ) ranging from 10.50 to $14.59 \%$, the percentage being higher for COI (19.41\%). For the COI protein coding gene, third codon positions were $54.19 \%$ variable, second codon positions were invariant and first codon positions were $4.47 \%$ variable. The $\mathrm{Ts} / \mathrm{Tv}$ ratio ranged between $2.61(16 \mathrm{~S})$ and 6.00 (COI) with 4.13 for $12 \mathrm{~S}$, and 2.63 for tRNA-valine. There was no evidence of sequence saturation in the analysed genes. For each gene sequence the goodness-of-fit test showed homogeneous base composition across taxa $(\mathrm{P}=1.00)$ and the partition homogeneity test showed no significant heterogeneity between genes ( $\mathrm{P}_{\text {ILD }}$ range from 0.15 to 1.00$)$, and although there is no generally accepted p-value for significant results, most authors agree to combine data when p-values are greater than 0.05 (Cristescu and Hebert, 2002; Russello and Amato, 2004).

As assessed in Carreras-Carbonell et al. (2005), two well-supported clades for $T$. tripteronotus (northern and southern) were found with posterior probabilities of $100 \%$. The southern clade belonging to $T$. tartessicum showed no well-supported structure pattern between different localities. However, the northern clade (T. tripteronotus) showed several well-supported subclades that could be related to defined geographical areas (e.g. Cyprus and Turkey), indicating some degree of isolation between different populations (Fig. 5).

Molecular divergence between $T$. tripteronotus and $T$. tartessicum ranges between $9.14 \%$ (COI) and $2.79 \%$ (tRNA-valine), with a mean value combining all genes of $6.89 \%$ (Table 2). No genetically and/or morphologically hybrid populations or individuals were found.

\section{DISCUSSION}

The new species is geographically distributed along the southern coast of Spain, from Cape La Nao 
TABLE 2. - Polymorphism and divergence within and between species, for each gene separately and all genes together (mean \pm SD percentage).

\begin{tabular}{|c|c|c|c|c|c|}
\hline & COI & $12 \mathrm{~S}$ & $16 \mathrm{~S}$ & tRNA-valine & All genes \\
\hline T. tripteronotus & $1.37 \pm 0.40$ & $1.24 \pm 0.30$ & $1.86 \pm 0.42$ & 0 & $1.33 \pm 0.03$ \\
\hline T. tartessicum & $0.25 \pm 0.08$ & $0.24 \pm 0.12$ & $0.29 \pm 0.05$ & 0 & $0.15 \pm 0.02$ \\
\hline T. tripteronotus vs. T. tartessicum & $9.14 \pm 2.01$ & $5.32 \pm 1.78$ & $6.72 \pm 0.95$ & $2.79 \pm 0$ & $6.89 \pm 0.73$ \\
\hline T. tripteronotus vs. T. delaisi & $13.58 \pm 3.92$ & $8.23 \pm 3.09$ & $10.85 \pm 2.81$ & $11.84 \pm 0$ & $11.03 \pm 2.60$ \\
\hline T. tartessicum vs. T. delaisi & $14.80 \pm 6.98$ & $6.87 \pm 3.44$ & $8.22 \pm 2.88$ & $8.70 \pm 0$ & $9.90 \pm 2.97$ \\
\hline T. tripteronotus vs. $P$. rouxi & $16.08 \pm 4.64$ & $24.97 \pm 9.31$ & $26.25 \pm 7.25$ & $18.56 \pm 0$ & $22.61 \pm 5.32$ \\
\hline T. tartessicum vs. P. rouxi & $18.49 \pm 8.72$ & $24.64 \pm 12.32$ & $24.59 \pm 8.61$ & $16.44 \pm 0$ & $22.35 \pm 6.71$ \\
\hline
\end{tabular}

(SP7) to the Gulf of Cadiz (SP12), the Balearic Islands (SP5 and SP6), and northern Africa, from Plage David (MC; Morocco, Atlantic Ocean) to Tunisia (TU1) (see Fig. 1). The eastern boundary in the distribution of the new species is unfortunately unknown. Some morphological characteristics (e.g. rays of the second dorsal fin and caudal bands) of the specimens collected in Israel by Zander and Heymer $(1970,1976)$ are closely related to those observed in the new species, suggesting the presence of $T$. tartessicum in that area. However, as we have mentioned above, these morphological characters are not constant, and unfortunately we could not analyse specimens from this locality. Future studies are recommended to confirm the taxonomic position of this material.

T. tripteronotus is restricted to the northern Mediterranean basin, including the NE coast of Spain (from SP4 to SP1), France (FR2 and also Corsica Is., FR1), Italy (IT1 and also Sicily Is., IT2), the Adriatic Sea (CR1-15 and MO1-2), Malta Is. (MA1), the Aegean Sea, including the coasts of Greece (GR3-5) and Turkey (TK3-6), as well as the Ciclades Islands (GR2) and Crete (GR1), the Marmara Sea (TK7), the Mediterranean Turkish coast and Cyprus (TK1-2 and CY1-2) (see Fig. 1).

The individuals from Nice (FR2) and Messina (IT2) were grouped within the northern clade of $T$. tripteronotus, suggesting that all specimens from these localities belonged to the species described by Risso (1810). Therefore, T. melaenocephalus, described by Cocco (1829), can be considered as a junior synonym of $T$. tripteronotus, in agreement with previous studies (e.g. Zander, 1986). The specimens from the Black Sea, originally identified as $T$. nikolskii (Maksimov, 1909) and synonymised with T. tripteronotus, could not be analysed. However, the presence of T. tripteronotus on the Aegean coasts of Greece and Turkey, as well as in the Marmara Sea, suggests that the specimens from the Black Sea may belong to T. tripteronotus or T. nikolskii, but not to the new species.
Our results confirm the validity of subtle morphological characters for distinguishing species of the genus Tripterygion, and the existence of a cryptic species, as occurs in other fish taxa (Gleeson et al., 1999; Henriques et al., 2002; Yamazaki et al., 2003). Nevertheless, the criteria used to designate distinct species based on molecular data are always controversial (Cracraft, 1989; Avise, 1994). The genetic divergence between $T$. tripteronotus and $T$. tartessicum is $9.14 \%$ for COI, $5.32 \%$ for $12 \mathrm{~S}$ and $6.72 \%$ for $16 \mathrm{~S}$, similar to the divergence observed between other fish taxa. Yamazaki et al. (2003), using COI, found a sequence difference of $9.10 \pm 0.36 \%$ between two cryptic species of brook lamprey. For $16 \mathrm{~S}$, genetic distances between congeneric species of the families Soleidae, Mullidae and Apogonidae range between 4.6 and 11.70\% (Tinti et al., 2000; Apostolidis et al., 2001; Mabuchi et al., 2003). Finally, for $12 \mathrm{~S}$ the mean genetic distance between congeneric species of the genus Coryphaenoides was $3.31 \%$ (Morita, 1999), 4\% within the genus Macullochella (Jerry et al., 2001) and a mean of 6.5\% within different blenniidae genera (Stepien et al., 1997). Henriques et al. (2002), in a revision of the genus Lepadogaster (Teleostei: Gobiesocidae), observed that the minimum distance between valid species was $3 \%$ at $12 \mathrm{~S}$ rRNA. Furthermore, Almada et al. (2005b), using $12 \mathrm{~S}$ and $16 \mathrm{~S}$ genes, showed that the genetic differences between clearly morphologically differentiated European blenniid species of the genus Parablennius and Lipophrys were even smaller (1.3-1.6\%). Within the genus Tripterygion, $T$. tripteronotus and T.tartessicum showed the smallest divergence, indicating a more recent speciation event (Carreras-Carbonell et al., 2005).

The estimated divergence time found between the two species was approximately $3.17 \mathrm{Myr}$ when the evolutionary rates of $0.81 \pm 0.23 \% / \mathrm{Myr}$ for $12 \mathrm{~S}$ and $1.10 \pm 0.23 \% / \mathrm{Myr}$ for $16 \mathrm{~S}$ inferred for the genus Tripterygion (Carreras-Carbonell et al., 2005) were applied. This divergence could be caused by the 
marine regressions during the Pliocene glaciations (2.7-3.6 Mya), when the sea level fell several meters. During the glaciations, a barrier could be formed between Cape La Nao (SP7) and the Balearic Islands (SP5 and SP6), acting as a separation between the two basins and allowing diversification between the two clades. However, we cannot discard the existence of a barrier elsewhere (e.g. the Gibraltar Strait) and a later expansion, the boundaries being the results of secondary contacts. Today, the low larval and adult dispersal capabilities of Tripterygion species (Heymer, 1977; Wirtz, 1978; Sabatés et al., 2003; Carreras-Carbonell et al., 2006) and the circulation regime that separates the northern from the southern basins (Send et al., 1999) could be maintaining the distribution areas of the two species non-overlapping.

\section{Key to the Mediterranean tripterygiids}

Modified from Zander (1986).

1a. Profile of head acute with an arch of about $60^{\circ}$; lips protruding; head mask of territorial males extending to breast; females and non-territorial males with marbled head; body permanently red. Tripterygion melanurus

1b. Profile of head more obtuse with an arch of about $70^{\circ}$; lips not protruding; head mask not extending to breast; females and non-territorial males without marbled head 2

2a. Last dark bar of body forms a distinct black spot on caudal peduncle with an extension onto base of caudal finrays; body of territorial males yellow, head mask not extending to tip of pectoral fins

2b. Last dark bar of body not forming an extension onto base of caudal fin; body of territorial males red; head mask extending to tip of pectoral fins.

3a. During the courtship males draw a figure-of- 8 swimming upwards into the water. Current distribution: Macaronesia

Tripterygion delaisi delaisi

3b. During the courtship males draw a figure-of- 8 swimming only on the bottom. Current distribution: Mediterranean Sea and Atlantic European coasts ....

Tripterygion delaisi xanthosoma

4a. Eyes large, head length less than 2.5 times orbit diameter (in individuals between 2 and $5 \mathrm{~cm}$ ) (Fig. 4) Tripterygion tartessicum 4b. Eyes moderately large, head length more than 2.5 times orbit diameter (in individuals between 2 and $5 \mathrm{~cm}$ ) (Fig. 4) Tripterygion tripteronotus

\section{ACKNOWLEDGEMENTS}

We are especially indebted to Ronald Fricke for providing specimens from the Staatliches Museum fuer Naturkunde (Stuttgart, SMNS) and his valuable comments on the manuscript. We also thank K. Clements and R.A. Patzner for their helpful suggestions. We are grateful to F. Fiorentino, J. Folch, P. Guidetti, B. Hereu, R.S. Santos, N. Sarpa and N. Sauleda for providing samples from different localities. We are indebted to C. Sarasquete, ICMANCSIC and the Junta de Andalucía staff for their valuable assistance in collecting the Holotype from the Gulf of Cádiz. We are also indebted to M. Bhaud for supplying some old manuscripts from the Observatoire Océanologique of Banyuls-sur-Mer. The illustrations were created by J. Macpherson. This research was supported by a pre-doctoral fellowship from the Ministerio de Educación, Cultura y Deporte to J.C. (AP2001-0225). The research was funded by projects CTM2004-05265 and BOS200305904 of the MCYT. The researchers are part of the SGR 2005SGR-00995 and 2005SGR-00277 of the Generalitat de Catalunya.

\section{REFERENCES}

Alfaro, M.E., S. Zoller and F. Lutzoni. - 2003. Bayes or Bootstrap? A simulation comparing the performance of Bayesian Markov Chain Monte Carlo sampling and bootstrapping in assessing phylogenetic confidence. Mol. Biol. Evol., 20: 255-266.

Almada, F., V.C. Almada, V. Domingues, A. Brito and R.S. Santos. - 2005a. Molecular validation of the specific status of Parablennius sanguinolentus and Parablennius parvicornis (Pisces: Blenniidae). Sci. Mar., 69: 519-532.

Almada, F., V.C. Almada, T. Guillemaud and P. Wirtz. - 2005b. Phylogenetic relationships of the North-eastern Atlantic and Mediterranean blenniids. Biol. J. Lin Soc., 86: 283-295.

Apostolidis, A.P., Z. Mamuris and C. Triantaphyllidis. - 2001. Phylogenetic relationships among four species of Mullidae (Perciformes) inferred from DNA sequences of mitochondrial cytochrome b and 16S rRNA genes. Bioch. Syst. Ecol., 29: 901-909.

Avise, J.C. - 1994. Molecular markers, natural history and evolution. Chapman and Hall, New York.

Carreras-Carbonell, J., E. Macpherson and M. Pascual. - 2005. Rapid radiation and cryptic speciation in Mediterranean triplefin blennies (Pisces: Tripterygiidae) combining multiple genes. Mol. Phylogenet. Evol., 37: 751-761.

Carreras-Carbonell, J., E. Macpherson and M. Pascual. - 2006. Population structure within and between subspecies of the Mediterranean triplefin fish Tripterygion delaisi revealed by highly polymorphic microsatellite loci. Mol. Ecol., 15: 3527-3529.

Cocco, A. - 1829. Su di alcuni nuovi pesci de'mari di Messina. 
Giorn. Sci Lett. Arti Sicilia, 26: 138-147.

Cracraft, J. - 1989. Speciation and its ontology: the empirical consequences of alternative species concepts for understanding patterns and processes of differentiation. In: D. Otte and J.A. Endler (eds.), Speciation and its Consequences, pp. 28-59, Sinauer Associates, Sunderland, Mass.

Cristescu, M.E.A and P.D.N. Hebert. - 2002. Phylogeny and adaptative radiation in the Onychopoda (Crustacea, Cladocera): evidence from multiple gene sequences. J. Evol. Biol., 15: 838-849.

Farris, J.S., M. Kallersjo, A.G. Kluge and C. Bult - 1994. Testing significance of incongruence. Cladistics, 10: 315-319.

Fricke, R., - 1997. Tripterygiid fishes of the western and central Pacific, with description of 15 new species, including an annotated checklist of world Tripterygiidae (Teleostei). Koenigstein, Koeltz Scientific Book, Theses Zoologicae 29.

Fricke, R., - 2002. Tripterygiid fishes of New Caledonia, with zoogeographical remarks. Environ. Biol. Fishes, 65: 175-198.

Gilles, A., A. Miquelis, J.P. Quignard and E. Faure. - 2000. Molecular phylogeography of western Mediterranean dusky grouper Epinephelus marginatus. Life Sci., 323: 195-205.

Gleeson, D.M., R.L.J. Howitt and N. Ling. - 1999. Genetic variation, population structure and cryptic species within the black mudfish, Neochanna diversus, and endemic galaxiid from New Zealand. Mol. Ecol., 8: 47-57.

Gysels, E.S., B. Hellemans, T. Patarnello and F.A.M. Volckaert. 2004. Current and historic gene flow of the sand goby Pomatoschistus minutus on the European Continental Shelf and in the Mediterranean Sea. Biol. J. Lin Soc., 83: 561-576.

Held, C. and J.W. Wagele. - 2005. Cryptic speciation in the giant Antarctic isopod Glyptonotus antarcticus (Isopoda: Valvifera: Chaetiliidae). Sci. Mar., 69:175-181.

Henriques, M., R. Lourenço, F. Almada, G. Calado, D. Gonçalves, T. Guillemaud, M.L. Cancela and V.C. Almada. - 2002. A revision of the status of Lepadogaster lepadogaster (Teleostei: Gobiesocidae): sympatric subspecies or a long misunderstood blend of species? Biol. J. Lin Soc., 76: 327-338.

Heymer, A. - 1977. Expériences subaquatiques sur les performances d'orientation et de retour au gite chez Tripterygion tripteronotus et Tripterygion xanthosoma (Blennioidei, Tripterygiidae). Vie Milieu, 3e sér. 27: 425-435.

Huelsenbeck, J.P. and F.R. Ronquist. - 2001. MrBayes: Bayesian inference of phylogenetic trees. Bioinformatics, 17: 754-755.

Hureau, J.C. and T. Monod. - 1973. Checklist of the fishes of the northeastern Atlantic and of the Mediterranean. Unesco, Paris.

Jerry, D.R., M.S. Elphinstone and P.R. Baverstock. - 2001. Phylogenetic relationships of Australian members of the Family Percichthyidae inferred from mitochondrial 12S rRNA sequence data. Mol. Phylogenet. Evol., 18: 335-347.

Knowlton, N. - 1993. Sibling species in the sea. Annu. Rev. Ecol. Syst., 24: 189-216.

Mabuchi, K., N. Okuda, T. Kokita and M. Nishida.-2003. Genetic comparison of two color-morphs of Apogon properutus from southern Japan. Ichthyol. Res., 50: 293-296.

Macpherson, E. - 1994. Substrate utilisation in a Mediterranean littoral fish community. Mar. Ecol. Prog. Ser., 114: 211-218.

Maksimov, N. - 1909. Zwei Tripterygium Arten aus dem Schwarzischen Meere. Soc. Nat. Charikow Trav., 42: 59-63.

Morita, T. - 1999. Molecular phylogenetic relationships of the deep-sea fish genus Coryphaenoides (Gadiformes: Macrouridae) based on mitochondrial DNA. Mol. Phylogenet. Evol., 13: 447-454.

Moyer, G.R., B.M. Burr and C. Krajewski. - 2004. Phylogenetic relationships of thorny catfishes (Siluriformes: Doradidae) inferred from molecular and morphological data. Zool. J. Linn. Soc., 140: 551-575.

Posada, D. and K.A. Crandall. - 1998. MODELTEST: testing the model of DNA substitution. Bioinformatics, 14: 817-818.

Risso, A. - 1810. Ichthyologie de Nice, ou histoire naturelle des poissons du département des Alpes Maritimes. F. Schoell, Paris. Ichthyol. Nice: i-xxxvi + 1-388.

Risso, A. - 1826. Histoire naturelle des principales productions de l'Europe méridionale et particulièrment de celles des environs de Nice et des Alpes maritimes. Paris et Strasbourg, 3: $\mathrm{XVI}+486$

Russello, M.A. and G. Amato. - 2004. A molecular phylogeny of Amazona: implications for Noetropical parrot biogeography, taxonomy and conservation. Mol. Phylogenet. Evol., 30: 421-437.

Sabatés, A., M. Zabala and A. Garcia-Rubies. - 2003. Larval fish communities in the Medes Islands marine reserve (north-west Mediterranean). J. Plankton Res., 25: 1035-1046.

Send, U., J. Font, G. Krahmann, C. Millot, M. Rhein and J. Tintore. - 1999. Recent advances in observing the physical oceanography of the western Mediterranean Sea. Prog. Oceanogr., 44: 37-64.

Stepien, C.A., A.K. Dillon, M.J. Brook, K.L. Chase and A.N. Hubers. - 1997. The evolution of Blennioid fishes based on analysis of mitochondrial $12 \mathrm{~S}$ rDNA. In: T.D. Kocher, C.A. Stepien (eds.), Molecular systematics of fishes, 245-270. Academic Press, San Diego.

Swofford, D.L. - 2001. PAUP*. Phylogenetic Analysis Using Parsimony (* and Other Methods), Version 4. Sinauer Associates, Sunderland, MA.

Tarjuelo, I., D. Posada, K.A. Crandall, M. Pascual and X. Turon.2001. Cryptic species of Clavellina (Ascidiacea) in two different habitats: harbours and rocky littoral zones in the northwestern Mediterranean. Mar. Biol., 139: 455-462.

Tinti, F. C. Piccinetti. S. Tommasini and M. Vallisneri. - 2000. Mitochondrial DNA variation, phylogenetic relationships and evolution of four Mediterranean genera of Soles (Soleidae, Pleuronectiformes). Mar. Biotechnol., 2: 274-284.

Wheeler, A. and J. Dunne. - 1975. Tripterygion atlanticus sp. nov. (Teleostei: Tripterygiidae), the first record of a tripterygiid fish in North-Western Europe. J. Fish Biol., 7: 639-649.

Wirtz, P. - 1978. The behaviour of the Mediterranean Tripterygion species (Pisces, Blennioidei). Z. Tierpsychol., 48: 142-174.

Wirtz, P. - 1980. A revision of the Eastern-Atlantic Tripterygiidae (Pisces, Blennioidei) and notes on some West African Blennioid fish. Cybium, 11: 83-101.

Yamazaki, Y., A. Goto and M. Nishida. - 2003. Mitochondrial DNA sequence divergence between two cryptic species of Lethenteron, with reference to an improved identification technique. J. Fish Biol., 62: 591-609.

Zander, C.D. and A. Heymer. - 1970. Tripterygion tripteronotus Risso 1810 und Tripterygion xanthosoma tartessicum, eine ökologische Speziation. Vie Milieu A, 21: 363-394.

Zander, C.D. and A. Heymer. - 1976. Morphologische und ökologische Untersuchungen an den speleophilen Schleimfischartigen Tripterygion melanurus Guichenot, 1850 und T. minor Kolombatovic, 1892 (Perciformes, Blennioidei, Tripterygiidae). Z. Zool. Syst. Evolut.-Forsch., 14: 41-59.

Zander, C.D. - 1986. Tripterygiidae In: P.J.P. Whitehead, M.L. Bauchot, J.C. Hureau, J. Nielsen, E. Tortonese (eds.), Fishes of the North-eastern Atlantic and the Mediterranean (Volume 3), 1118-1121. UNESCO, Paris.

Zander, C.D. - 2004. Ecology meets genetics-niche occupation as a factor of evolution interpreted by KOSSWIG's concepts. Mitt. Hamburg. Zool. Mus. Inst., 101: 131-147.

Scient. ed.: M.P. Olivar.

Received October 25, 2005. Accepted September 28, 2006.

Published online February 8, 2007. 